\title{
Erratum to: The Java Image Science Toolkit (JIST) for Rapid Prototyping and Publishing of Neuroimaging Software
}

Blake C. Lucas • John A. Bogovic • Aaron Carass •

Pierre-Louis Bazin • Jerry L. Prince • Dzung L. Pham •

Bennett A. Landman

Published online: 9 March 2010

(C) Springer Science+Business Media, LLC 2010

\section{Erratum to: Neuroinform}

DOI 10.1007/s12021-009-9061-2

The original article provided incomplete information on funding. Blake Lucas was supported in part by the Intramural Research Program, NIA, NIH.

The online version of the original article can be found at http://dx.doi. org/10.1007/s12021-009-9061-2.

B. C. Lucas · J. A. Bogovic · A. Carass · J. L. Prince Department of Electrical and Computer Engineering, Johns Hopkins University,

Baltimore, MD, USA

\section{B. C. Lucas}

National Institute on Aging, National Institute of Health,

Baltimore, MD, USA

P.-L. Bazin · J. L. Prince • D. L. Pham

Department of Radiology and Radiological Science,

Johns Hopkins University,

Baltimore, MD, USA

J. L. Prince $\cdot$ B. A. Landman $(\triangle)$

Department of Biomedical Engineering,

Johns Hopkins University,

Baltimore, MD, USA

e-mail: bennett.landman@vanderbilt.edu

B. A. Landman

Department of Electrical Engineering, Vanderbilt University,

Nashville, TN, USA

B. A. Landman

Department of Radiology and Radiological Sciences,

Vanderbilt University,

Nashville, TN, USA 